\title{
SPLIT REDUCTIONS OF SIMPLE ABELIAN VARIETIES
}

\author{
JEFFREY D. AChTER
}

\begin{abstract}
Consider an absolutely simple abelian variety $X$ over a number field $K$. We show that if the absolute endomorphism ring of $X$ is commutative and satisfies certain parity conditions, then $X_{\mathfrak{p}}$ is absolutely simple for almost all primes $\mathfrak{p}$. Conversely, if the absolute endomorphism ring of $X$ is noncommutative, then $X_{\mathfrak{p}}$ is reducible for $\mathfrak{p}$ in a set of positive density.
\end{abstract}

An absolutely simple abelian variety over a number field may or may not have absolutely simple reduction almost everywhere. On one hand, let $K=\mathbb{Q}\left(\zeta_{5}\right)$, and let $X$ be the Jacobian of the hyperelliptic curve with affine model

$$
t^{2}=s(s-1)\left(s-1-\zeta_{5}\right)\left(s-1-\zeta_{5}-\zeta_{5}^{2}\right)\left(s-1-\zeta_{5}-\zeta_{5}^{2}-\zeta_{5}^{3}\right),
$$

considered as an abelian surface over $K$. Then $X$ is absolutely simple [13, p.648] and has ordinary reduction at a set of primes $\mathfrak{p}$ of density one [12, Prop. 1.13]; at such primes $X_{\mathfrak{p}}$ is absolutely simple.

On the other hand, let $Y$ be the Jacobian of the hyperelliptic curve with affine model

$$
t^{2}=s^{6}-12 s^{5}+9 s^{4}-32 s^{3}+3 s^{2}+18 s+3,
$$

considered as an abelian surface over $L=\mathbb{Q}(\sqrt{2}, \sqrt{-3})$. Then $Y$ is absolutely simple [3. Thm. 6.1], but $Y_{\mathfrak{q}}$ is reducible for each prime $\mathfrak{q}$ of good reduction. (The conclusions about the simplicity of $X_{\mathfrak{p}}$ and the reducibility of $Y_{\mathfrak{q}}$ follow from Tate's description 25] of the endomorphism rings of abelian varieties over finite fields.)

Note that $\operatorname{End}_{K}(X) \otimes \mathbb{Q}$ is the cyclotomic field $\mathbb{Q}\left(\zeta_{5}\right)$, while $\operatorname{End}_{L}(Y) \otimes \mathbb{Q}$ is an indefinite quaternion algebra over $\mathbb{Q}$. Murty and Patankar study the splitting behavior of abelian varieties over number fields, and advance the following conjecture:

Conjecture. [20, Conj. 5.1] Let $X / K$ be an absolutely simple abelian variety over a number field. The set of primes of $K$ where $X$ splits has positive density if and only if $\operatorname{End}_{\bar{K}}(X)$ is noncommutative.

(A similar question has been raised by Kowalski; see [14, Rem. 3.9].) The present paper proves this conjecture under certain parity and signature conditions on $\operatorname{End}(X)$.

The first main result states that a member of a large class of abelian varieties with commutative endomorphism ring has absolutely simple reduction almost everywhere. (Throughout this paper, "almost everywhere" means for a set of primes of density one.)

Theorem A. Let $X / K$ be an absolutely simple abelian variety over a number field. Suppose that either

(i) $\operatorname{End}_{\bar{K}}(X) \otimes \mathbb{Q} \cong F$ a totally real field, and $\operatorname{dim} X /[F: \mathbb{Q}]$ is odd; or

Received by the editors May 21, 2008. 
(ii) $\operatorname{End}_{\bar{K}}(X) \otimes \mathbb{Q} \cong E$ a totally imaginary field, and the action of $E$ on $X$ is not special.

Then for almost every prime $\mathfrak{p}, X_{\mathfrak{p}}$ is absolutely simple.

(The notion of "not special" is discussed in Section 4 , it is satisfied if, for instance, $\operatorname{dim} X$ is prime.) Conversely, the second main result shows that abelian varieties with noncommutative endomorphism ring have split reduction at a set of primes of positive density.

Theorem B. Suppose $X / K$ is an absolutely simple abelian variety over a number field, and that $\operatorname{End}_{\bar{K}}(X)$ is noncommutative.

(i) For $\mathfrak{p}$ in a set of positive density, $X_{\mathfrak{p}}$ is absolutely reducible.

(ii) Suppose $\operatorname{End}_{\bar{K}}(X) \otimes \mathbb{Q}$ is an indefinite quaternion algebra over a totally real field $F$, and that $\operatorname{dim} X / 2[F: \mathbb{Q}]$ is odd. For $\mathfrak{p}$ in a set of positive density, $X_{\mathfrak{p}}$ is geometrically isogenous to the self-product of an absolutely simple abelian variety.

Moreover, there is a finite extension of $K$ such that the set of primes $\mathfrak{p}$ in Theorem B actually has density one.

Special cases of these results are already known. The case of Theorem A(i) in which $\operatorname{End}(X) \cong \mathbb{Z}$ is due to Chavdarov [5, Cor. 6.10]; see also the related work of Chai and Oort 4. An abelian surface over a finite field with noncommutative endomorphism ring is absolutely reducible [19, p.261], and the special case of Theorem B(i) in which $F=\mathbb{Q}$ and $\operatorname{dim} X=2$ is apparently well-known [1, Cor. 2]. More recently, Murty and Patankar have shown that if $X$ is either an abelian variety of CM type [20, Thm. $3.1]$, or a modular abelian variety with commutative absolute endomorphism ring [20, Thm. 4.1], then $X$ has simple reduction almost everywhere.

Ellenberg et al. 10 have addressed a related problem for families of abelian varieties over a number field. Specifically, they consider the relative Jacobian of a family of hyperelliptic curves $y^{2}=f(x)(x-t)$ over $K[t]$, and show that for all but finitely many specializations of $t$ the resulting abelian variety is simple.

The proof of Theorem B uses the fact that, if $\operatorname{End}_{\bar{K}}(X)$ is noncommutative, then the Tate module $T_{\ell}(X)$ is a direct sum of copies of the same representation of $\mathrm{Gal}(K)$. This, in turn, follows from the fact [18] that the first homology of $X$, as a representation of the Lefschetz group, is isotypic but not irreducible.

The proof of Theorem A is more involved, and uses the Chebotarev theorem and the observation that if the Frobenius at $\mathfrak{p}$ acts irreducibly on the $\ell$-torsion for some $\ell$, then $X_{\mathfrak{p}}$ is simple. This approach was used by Chavdarov in [5, Cor. 6.10] in the special case where the image of $\operatorname{Gal}(K)$, acting on each $X_{\ell}$, is the group of symplectic similitudes $\mathrm{GSp}_{2 g}(\mathbb{Z} / \ell)$. We give a more detailed outline of this strategy in the following example, which gives a quick proof of a special (but typical) case of [20, Thm. 3.1]. Let $E$ be a totally imaginary extension of $\mathbb{Q}$ of degree $2 g$. Suppose that $X / K$ is an absolutely simple $g$-dimensional abelian variety with $\operatorname{End}_{K}(X)=\operatorname{End}_{\bar{K}}(X) \cong \mathcal{O}_{E}$. Further suppose that the CM type of $E$ is nondegenerate in the sense of [15]. For each rational prime $\ell$ there are Galois representations $\rho_{X / K, \mathbb{Z}_{\ell}}: \operatorname{Gal}(K) \rightarrow \operatorname{Aut}\left(T_{\ell}(X)\right) \cong \mathrm{GL}_{2 g}\left(\mathbb{Z}_{\ell}\right)$ and $\rho_{X / K, \ell}: \operatorname{Gal}(K) \rightarrow \operatorname{Aut}\left(X_{\ell}\right) \cong \mathrm{GL}_{2 g}(\mathbb{Z} / \ell)$. There is a set of rational primes $\mathbb{L}$ (containing all but finitely many primes) such that if $\ell_{1}, \cdots, \ell_{r}$ are distinct primes 
in $\mathbb{L}$, then the image of $\operatorname{Gal}(K)$ under the product representation $\times_{1 \leq i \leq r} \rho_{X / K, \ell_{i}}$ is $\times_{1 \leq i \leq r}\left(\mathcal{O}_{E} \otimes \mathbb{Z} / \ell_{i}\right)^{\times}$(e.g., 22] $)$.

Let $\ell$ be any prime at which $E$ is inert, so that $\left(\mathcal{O}_{E} \otimes \mathbb{Z} / \ell\right)^{\times} \cong \mathbb{F}_{\ell^{2} g}^{\times}$. Let $I_{\ell}$ be the set of elements of $\mathbb{F}_{\ell^{2} g}^{\times}$which are members of some proper subfield of $\mathbb{F}_{\ell^{2 g}}$. Note that if $\mathbb{F}_{\ell^{2 g}}$ is considered as a vector space over $\mathbb{Z} / \ell$, then elements of $I_{\ell}$ are precisely the elements of $\mathbb{F}_{\ell^{2 g}}^{\times}$which acts reducibly on $\mathbb{F}_{\ell^{2 g}}$. There exists a constant $C<1$ such that for all $\ell$ inert in $E$, we have $\left|I_{\ell}\right| /\left|\mathbb{F}_{\ell^{2 g}}^{\times}\right|<C$.

Let $M(X / K)$ be the set of (finite) primes of $K$ where $X$ has good reduction, and let $R(X / K)$ be the set of primes $\mathfrak{p}$ of good reduction for which $X_{\mathfrak{p}}$ is reducible. Suppose $\mathfrak{p} \in M(X / K)$, and let $\sigma_{\mathfrak{p}} \in \operatorname{Gal}(K)$ be a Frobenius element at $\mathfrak{p}$. Let $\ell$ be a rational prime relatively prime to $\mathfrak{p}$. The Frobenius endomorphism of $X_{\mathfrak{p}}$ acts as $\rho_{X / K, \mathbb{Z}_{\ell}}\left(\sigma_{\mathfrak{p}}\right)$ on $T_{\ell}\left(X_{\mathfrak{p}}\right) \cong T_{\ell}(X)$. If $X_{\mathfrak{p}}$ is not simple, then the $\operatorname{Gal}(\kappa(\mathfrak{p}))$-module $T_{\ell}\left(X_{\mathfrak{p}}\right)$ is reducible, and in particular $\rho_{X / K, \ell}\left(\sigma_{\mathfrak{p}}\right)$ acts reducibly on $X_{\mathfrak{p}, \ell}:=X_{\mathfrak{p}}[\ell](\overline{\kappa(\mathfrak{p})})$. Therefore, if there exists one prime $\ell$ such that $\rho_{X / K, \ell}\left(\sigma_{\mathfrak{p}}\right)$ acts irreducibly on $X_{\mathfrak{p}, \ell}$, then $X_{\mathfrak{p}}$ is simple.

So, let $\ell_{1}, \cdots, \ell_{r}$ be distinct primes in $\mathbb{L}$ at which $E$ is inert. Let $R\left(X / K ; \ell_{1}, \cdots, \ell_{r}\right)$ $\subset M(X / K)$ be the set of primes $\mathfrak{p}$ such that for each $1 \leq i \leq r, \rho_{X / K, \ell_{i}}\left(\sigma_{\mathfrak{p}}\right) \in I_{\ell_{i}}$. Then $R(X / K) \subseteq R\left(X / K ; \ell_{1}, \cdots, \ell_{r}\right)$. By the Chebotarev theorem, the density of $R\left(X / K ; \ell_{1}, \cdots, \ell_{r}\right)$ is $\prod_{i=1}^{r}\left|I_{\ell_{i}}\right| /\left|\mathbb{F}_{\ell_{i}^{2 g}}^{\times}\right|<C^{r}$. Since $C<1$ and we may take an arbitrarily large set of rational primes inert in $E$, the density of $R(X / K)$ is zero, and the density of its complement is therefore one.

Generalizing this argument to other abelian varieties with commutative absolute endomorphism ring requires calculating the image of the Galois representations $\rho_{X / K, \mathbb{Z}_{\ell}}$, which is conjecturally described by the Mumford-Tate conjecture (see Section 3); showing that a positive proportion of elements of $\rho_{X / K, \ell}(\operatorname{Gal}(K))$ act irreducibly on the Tate module (Section 1); and axiomatizing the foregoing argument (Section 2.

Quite recently, Banaszak et al. have extended the methods of 2 to abelian varieties of type III, which allows an extension of Theorem 5.4 to the case of definite quaternion algebras. Also, Zywina points out that sieve methods (e.g., those of [28]) can be used to make the density one statements in Section 4 more explicit. I will explain both of these developments in detail elsewhere.

\section{Groups of Lie type}

If $B$ is a finite $A$-algebra, let $\mathbf{R}_{B / A}$ denote Weil's restriction of scalars functor.

Group schemes $G / \mathbb{Z}[1 / \Delta]$ of the following forms arise as the images of Galois representations considered here:

(A) There exist a totally imaginary field $E$ with maximal totally real subfield $F$; an $\mathcal{O}_{E}[1 / \Delta]$-module $V$ which is free of rank $2 r$ over $\mathcal{O}_{F}[1 / \Delta]$; and an $\mathcal{O}_{E}[1 / \Delta]$-Hermitian pairing $\langle\cdot, \cdot\rangle$ on $V$; such that $G$ is the Weil restriction $G=\mathbf{R}_{\mathcal{O}_{E}[1 / \Delta] / \mathbb{Z}[1 / \Delta]} \mathrm{GU}(V,\langle\cdot, \cdot\rangle)$. Let $Z=E$.

(C) There exist a totally real field $F$; a free $\mathcal{O}_{F}[1 / \Delta]$-module $V$ of rank $2 r$; and an $\mathcal{O}_{F}[1 / \Delta]$-linear symplectic pairing $\langle\cdot, \cdot\rangle$ on $V$; such that $G=$ $\mathbf{R}_{\mathcal{O}_{F}[1 / \Delta] / \mathbb{Z}[1 / \Delta]} \operatorname{GSp}(V,\langle\cdot, \cdot\rangle)$. Let $Z=F$. 
The center $Z G$ of $G$ satisfies $Z G(\mathbb{Z}[1 / \Delta]) \cong \mathcal{O}_{Z}[1 / \Delta]^{\times}$. The adjoint form of $G$ is $G^{\text {ad }}:=G / Z G$. For each $\ell$ inert in $Z$, let $T_{\ell}^{\text {an }} \subset G(\mathbb{Z} / \ell)$ be a maximally anisotropic maximal torus.

In case [A], the derived group of $G$ is $G^{\text {der }}=\mathbf{R}_{\mathcal{O}_{E}[1 / \Delta] / \mathbb{Z}[1 / \Delta]} \mathrm{SU}(V,\langle\cdot, \cdot\rangle)$. Note that $G(\mathbb{Z} / \ell) \cong \mathrm{GU}\left(V \otimes \mathcal{O}_{E} / \ell,\langle\cdot, \cdot\rangle\right)$, and $G^{\operatorname{der}}(\mathbb{Z} / \ell) \cong \mathrm{SU}\left(V \otimes \mathcal{O}_{E} / \ell,\langle\cdot, \cdot\rangle\right)$. In particular, if $\ell$ is a rational prime inert in $E$, then $G^{\text {der }}(\mathbb{Z} / \ell) \cong \operatorname{SU}_{r}\left(\mathcal{O}_{E} / \ell\right)$. Moreover, if $r$ is odd, then $T_{\ell}^{\text {an }}$ acts irreducibly on $V \otimes \mathbb{Z} / \ell$; while if $r$ is even, then $T_{\ell}^{\text {an }}$ stabilizes two subspaces which are in duality with each other.

In case (C), the derived group of $G$ is $G^{\text {der }}=\mathbf{R}_{\mathcal{O}_{F}[1 / \Delta] / \mathbb{Z}[1 / \Delta]} \operatorname{Sp}(V,\langle\cdot, \cdot\rangle)$. Note that $G(\mathbb{Z} / \ell) \cong \operatorname{GSp}\left(V \otimes \mathcal{O}_{F} / \ell,\langle\cdot, \cdot\rangle\right)$ and $G^{\operatorname{der}}(\mathbb{Z} / \ell) \cong \operatorname{Sp}\left(V \otimes \mathcal{O}_{F} / \ell,\langle\cdot, \cdot\rangle\right)$. In particular, if $\ell$ is a rational prime inert in $F$, then $G^{\operatorname{der}}(\mathbb{Z} / \ell) \cong \operatorname{Sp}_{2 r}\left(\mathcal{O}_{F} / \ell\right)$. Moreover, $T_{\ell}^{\text {an }}$ acts irreducibly on $V \otimes \mathbb{Z} / \ell$.

Let $G$ be a group scheme over $\mathbb{Z}[1 / \Delta]$. For a rational prime $\ell \nmid \Delta$, let $J_{\ell}(G)$ be the set of all $x \in G(\mathbb{Z} / \ell)$ for which the connected component of the centralizer of $x$ is a torus which is maximally anistropic. Let $I_{\ell}(G)$ be the complement $G(\mathbb{Z} / \ell)-J_{\ell}(G)$. Let $J_{\ell, m}(G)$ be the set of $x$ such that $x^{m} \in J_{\ell}(G)$, and let $I_{\ell, m}(G)$ be its complement. Each of these sets is stable under conjugation. For $G$ of type $(\mathrm{A})$ or $(\mathrm{C}), x \in J_{\ell}(G)$ if and only if $x$ is $G(\mathbb{Z} / \ell)$-conjugate to a regular element of $T_{\ell}^{\text {an }}$.

Say that an abstract group $H_{\ell}$ is of type $G(\mathbb{Z} / \ell)$ if there are inclusions $G^{\text {der }}(\mathbb{Z} / \ell) \subseteq$ $H_{\ell} \subseteq G(\mathbb{Z} / \ell)$. For such a group $H_{\ell}$, let $I_{\ell, m}\left(H_{\ell}\right)=H_{\ell} \cap I_{\ell, m}(G)$, and let $J_{\ell, m}\left(H_{\ell}\right)=$ $H_{\ell} \cap J_{\ell, m}(G)$.

Lemma 1.1. Suppose $G / \mathbb{Z}[1 / \Delta]$ is a group of type $(\mathrm{A}$ or $(\mathrm{C})$, and let $m$ be a natural number. There exists a constant $C=C(m, G)$ such that if $\ell$ is inert in $Z$ and sufficiently large, and if $H_{\ell}$ is of type $G(\mathbb{Z} / \ell)$, then $\left|I_{\ell, m}\left(H_{\ell}\right)\right| /\left|H_{\ell}\right|<C$.

Proof. First, for each $m \in \mathbb{N}$ we show the existence of a positive constant $D_{0}(m, G)$ such that for all sufficiently large $\ell$ inert in $Z,\left|J_{\ell, m}(G)\right| /|G(\mathbb{Z} / \ell)|>D_{0}(m, G)$. Subsequently, we show how to deduce a uniform statement for all $H_{\ell}$ of type $G(\mathbb{Z} / \ell)$.

Let $T_{\ell}^{*}$ be the set of regular elements of $T_{\ell}^{\text {an }}$, and let $T_{\ell, m}^{*}$ be the set of $x \in T_{\ell}^{\text {an }}$ such that $x^{m} \in T_{\ell}^{*}$. There are monic polynomials $f$ and $f^{*}$ of the same degree such that $\left|T_{\ell}^{\text {an }}\right|=f(\ell)$ and $\left|T_{\ell}^{*}\right|=f^{*}(\ell)$ [11. (In fact, 11] works out the analogous polynomials for $\left|T_{\ell}^{*} \cap G^{\prime}(\mathbb{Z} / \ell)\right|$, but the result for $G(\mathbb{Z} / \ell)$ itself follows immediately.) Therefore, there exists a constant $B$ such that, if $\ell \gg 0$, then $\left|T_{\ell}^{*}\right| /\left|T_{\ell}^{\text {an }}\right|>1-B / \ell$. By considering the fibers of the $m^{t h}$ power map, we see that $\left|T_{\ell, m}^{*}\right| /\left|T_{\ell}^{\text {an }}\right|>1-m B / \ell$.

An element of $G(\mathbb{Z} / \ell)$ is in $J_{\ell, m}(G)$ if and only if it is conjugate to an element of $T_{\ell, m}^{*}$. The normalizer $N_{\ell}=N_{G(\mathbb{Z} / \ell)}\left(T_{\ell}^{\text {an }}\right)$ is an extension of a finite group $W$ by $T_{\ell}^{\text {an }}$; the group $W$ depends on $G$, but not on $\ell$. Moreover, $T_{\ell, m}^{*}$ is stable under the action of $N_{\ell}$. We obtain the estimate

$$
\begin{aligned}
\frac{\left|J_{\ell, m}(G)\right|}{|G(\mathbb{Z} / \ell)|} & =\frac{1}{|G(\mathbb{Z} / \ell)|}\left(\frac{|G(\mathbb{Z} / \ell)|}{\left|N_{\ell}\right|}\left|T_{\ell, m}^{*}\right|\right) \\
& =\frac{1}{|W|} \frac{\left|T_{\ell, m}^{*}\right|}{\left|T_{\ell}^{\text {an }}\right|}>\frac{1}{|W|}\left(1-\frac{m B}{\ell}\right) .
\end{aligned}
$$

This shows the existence of $D_{0}(m, G)$ with the desired properties. Membership in $J_{\ell, m}(G)$ is well-defined on cosets modulo the center of $G$. Therefore, the proportion 
of elements of $G^{\text {ad }}(\mathbb{Z} / \ell)$ which are (represented by) elements whose $m^{\text {th }}$ power is maximally anisotropic is also at least $D_{0}(m, G)$.

Now let $H_{\ell}$ be any group of type $G(\mathbb{Z} / \ell)$, and let $H_{\ell}^{\text {ad }}=H_{\ell} /\left(Z G(\mathbb{Z} / \ell) \cap H_{\ell}\right)$. There is an inclusion of groups $H_{\ell}^{\text {ad }} \hookrightarrow G^{\text {ad }}(\mathbb{Z} / \ell)$, with cokernel a finite cyclic group whose order $n$ divides the rank of $G$.

Suppose $x \in J_{\ell, m n}(G)$. The equivalence class of $x^{n}$ modulo the center is represented by an element $h$ of $H_{\ell}$. Moreover, for such an $h, h^{m} \equiv x^{m n} \bmod Z G(\mathbb{Z} / \ell)$ is maximally anisotropic modulo the center. The elements of $G^{\text {ad }}(\mathbb{Z} / \ell)$ which are maximally anisotropic give rise to at least $\frac{1}{n} D_{0}(m n, G)\left|G^{\text {ad }}(\mathbb{Z} / \ell)\right|$ distinct maximally anisotropic elements of $H_{\ell}^{\text {ad }}$. Let $D(m, G)=\min \left\{\frac{1}{n} D_{0}(m n, G): n \mid \operatorname{rank}(G)\right\}$. Then one may take $1-D(m, G)$ for $C(m, G)$ in the statement of Lemma 1.1 .

Remark 1.2. For groups of type $(\mathrm{C})$, the case $m=1$ and $H_{\ell}=G(\mathbb{Z} / \ell)$ of Lemma 1.1 is proved in [5, Cor. 3.6].

Lemma 1.3. Let $G / \mathbb{Z}[1 / \Delta]$ be a group scheme. Suppose that either $G$ is of type $(A)$ with $r \geq 2$ or that $G$ is of type (C). Let $\ell_{1}, \cdots, \ell_{m}$ be distinct rational primes which are inert in $Z$. Let $H$ be a subgroup of $G^{\mathrm{der}}\left(\mathbb{Z} /\left(\prod \ell_{i}\right)\right)$ such that for each $i$, the composition $H \hookrightarrow G^{\mathrm{der}}\left(\mathbb{Z} /\left(\prod \ell_{i}\right)\right) \rightarrow G^{\mathrm{der}}\left(\mathbb{Z} / \ell_{i}\right)$ is surjective. Then $H=G^{\operatorname{der}}\left(\mathbb{Z} /\left(\prod \ell_{i}\right)\right)$.

Proof. This is Goursat's lemma [21, p. 793]; see also [5, Prop. 5.1]. The hypothesis guarantees that the adjoint groups $G^{\text {ad }}\left(\mathbb{Z} / \ell_{i}\right)$ are distinct nonabelian simple groups.

Lemma 1.4. Let $r \in \mathbb{N}$ and let $\mathbb{F}$ be a finite field, with $\mathbb{F} \notin\left\{\mathbb{F}_{2}, \mathbb{F}_{4}, \mathbb{F}_{3}, \mathbb{F}_{9}\right\}$. Suppose that either $G$ is $\mathrm{GU}_{r} / \mathbb{F}$ and $r \geq 2$ or that $G$ is $\mathrm{GSp}_{2 r} / \mathbb{F}$. Let $G^{\text {der }}$ be the derived group of $G$, let $G^{\text {ad }}$ be the adjoint form of $G$, and let $\alpha: G \rightarrow G^{\text {ad }}$ and $\beta: G^{\text {der }} \rightarrow G^{\text {ad }}$ be the canonical projections. Let $H \subset G(\mathbb{F})$ be a subgroup. If $\beta^{-1}(\alpha(H))=G^{\mathrm{der}}(\mathbb{F})$, then $H$ contains $G^{\mathrm{der}}(\mathbb{F})$.

Proof. This is standard; the hypothesis on $\mathbb{F}$ rules out exceptional cases.

\section{Abelian varieties}

Let $X / k$ be a principally polarized abelian variety over a field $k$. For each rational prime $\ell$ invertible in $k$, let $T_{\ell}(X)$ be the $\ell$-adic Tate module of $X$, and let $X_{\ell}:=$ $X[\ell](\bar{k})=T_{\ell}(X) / \ell T_{\ell}(X)$. Then $T_{\ell} X$ and $X_{\ell}$ come equipped with an action by Gal $(k)$. Let $\rho_{X / k, \mathbb{Z}_{\ell}}: \operatorname{Gal}(k) \rightarrow \operatorname{Aut}\left(T_{\ell}(X)\right)$ and $\rho_{X / k, \ell}: \operatorname{Gal}(k) \rightarrow \operatorname{Aut}\left(X_{\ell}\right)$ be the associated representations, with respective images $H_{X / k, \mathbb{Z}_{\ell}}$ and $H_{X / k, \ell}$. Let $H_{X / k, \mathbb{Q}_{\ell}}$ be the Zariski closure of $H_{X / k, \mathbb{Z}_{\ell}}$ in $\operatorname{Aut}\left(T_{\ell}(X) \otimes \mathbb{Q}\right)$.

Suppose $X$ is simple. Then the endomorphism algebra $D(X)=\operatorname{End}(X) \otimes \mathbb{Q}$ is a central simple algebra over a number field $E(X)$ with positive involution. Let $F(X) \subseteq$ $E(X)$ be the subfield fixed by the involution. Then $F(X)$ is a totally real field, and either $E(X)=F(X)$ or $E(X)$ is a totally imaginary quadratic extension of $F(X)$. Let $f(X)=[F(X): \mathbb{Q}]$, let $e(X)=[E(X): \mathbb{Q}]$, and let $d(X)=\sqrt{[D(X): E(X)]}$.

If $X$ and $Y$ are isogenous abelian varieties, write $X \sim Y$. 
If $K$ is a number field, let $M_{K}$ be the set of (finite) primes of $K$; if $\mathfrak{p} \in M_{K}$, denote its residue field by $\kappa(\mathfrak{p})$. Suppose $X / K$ is an absolutely simple abelian variety. As in the introduction, let $M(X / K) \subset M_{K}$ be the set of primes of good reduction of $X$. It is convenient to distinguish the following subsets of $M(X / K)$ :

- $S(X / K)=\left\{\mathfrak{p} \in M(X / K): X_{\mathfrak{p}}\right.$ is simple $\}$

- $S^{*}(X / K)=\left\{\mathfrak{p} \in M(X / K): X_{\mathfrak{p}}\right.$ is absolutely simple $\}$;

- $R(X / K)=\left\{\mathfrak{p} \in M(X / K): X_{\mathfrak{p}}\right.$ is reducible $\}$

- $R^{*}(X / K)=\left\{\mathfrak{p} \in M(X / K): X_{\mathfrak{p}}\right.$ is absolutely reducible $\}$.

Then $R(X / K)$ is the complement of $S(X / K) ; R^{*}(X / K)$ is the complement of $S^{*}(X / K) ; S^{*}(X / K) \subset S(X / K)$; and $R^{*}(X / K) \supset R(X / K)$. In this notation, 20, Conj. 5.1] states that $S(X / K)$ has density one if and only if $\operatorname{End}_{\bar{K}}(A)$ is commutative.

Many attributes of $X_{\bar{K}}$, the base change of $X$ to an algebraic closure of $K$, are already detectable over a finite extension of $K$. Consider the following condition on an abelian variety $X$ over a number field $K$ and a finite, Galois extension $K^{\prime} / K$ :

$\operatorname{End}_{K^{\prime}}(X)=\operatorname{End}_{\bar{K}}(X)$; for all but finitely many $\mathfrak{p} \in M(X / K)$, if $\mathfrak{p}^{\prime} \in M\left(X / K^{\prime}\right)$ is a prime which divides $\mathfrak{p}$, and if $X_{\mathfrak{p}^{\prime}}$ is simple, then $X_{\mathfrak{p}}$ is absolutely simple; and $H_{X / K^{\prime}, \mathbb{Q}_{\ell}}$ is connected for each rational prime $\ell$.

Lemma 2.1. Let $X / K$ be an abelian variety over a number field. Fix a natural number $n \geq 5$, and let $K^{\prime} / K$ be a finite, Galois extension which contains the field of definition of all $n$-torsion points of $X$. Then $\left(X / K, K^{\prime}\right)$ satisfies (2.1).

Proof. There are three conditions in 2.1). The first follows from Silverberg's criterion [23. Thm. 2.4]. The second follows from this and the fact that, if $\mathfrak{p}$ is relatively prime to $n$, then $X[n]\left(K^{\prime}\right) \hookrightarrow X_{\mathfrak{p}^{\prime}}[n]\left(\kappa\left(\mathfrak{p}^{\prime}\right)\right)$. The final condition is [24, Thm. 4.6].

In Lemma 2.1, if one insists that $n$ be divisible by two distinct primes $n_{1}$ and $n_{2}$, each of which is at least five, then the second condition of (2.1) holds for all primes $\mathfrak{p} \in M(X / K)$.

Throughout this paper Lemma 2.1 will be used, often implicitly, to show the existence of an extension $K^{\prime} / K$ such that $\left(X / K, K^{\prime}\right)$ satisfies 2.1).

We will often work with an abelian variety $X / K$, a group scheme $G / \mathbb{Z}[1 / \Delta]$, and an infinite set of rational primes $\mathbb{L} \subset M_{\mathbb{Q}}$ relatively prime to $\Delta$ which satisfy the following hypotheses:

The abelian variety $X$ is absolutely simple. For each $\ell \in M_{\mathbb{Q}}$,

$H_{X / K, \ell}$ is isomorphic to a subgroup of $G(\mathbb{Z} / \ell)$. For each $\ell \in \mathbb{L}$, $H_{X / K, \ell}$ is of type $G(\mathbb{Z} / \ell)$. For each finite subset $A \subset \mathbb{L}$, the image of $\operatorname{Gal}(K)$ under $\times_{\ell \in A} \rho_{X, \ell}$ is $\times_{\ell \in A} H_{X / K, \ell}$.

If $(X / K, G / \mathbb{Z}[1 / \Delta], \mathbb{L})$ satisfies 2.2 , and if $A \subset M_{\mathbb{Q}}$ is any set of primes, let $I(X / K ; G ; A) \subset M(X / K)$ be the set of primes $\mathfrak{p}$ such that for each $\ell \in A$ and each Frobenius element $\sigma_{\mathfrak{p}} \in \operatorname{Gal}(K)$ at $\mathfrak{p}, \rho_{X, \ell}\left(\sigma_{\mathfrak{p}}\right) \in I_{\ell}(G)$. Its complement $J(X / K ; G ; A)$ is the set of primes $\mathfrak{p} \in M(X / K)$ for which there exists some prime $\ell \in A$ such that $\rho_{X, \ell}\left(\sigma_{\mathfrak{p}}\right) \in J_{\ell}(G)$.

Let $I(X / K ; G)=I\left(X / K ; G ; M_{\mathbb{Q}}\right)$ and let $J(X / K ; G)=J\left(X / K ; G ; M_{\mathbb{Q}}\right)$. Note that for any $A \subset M_{\mathbb{Q}}, I(X / K ; G) \subseteq I(X / K ; G ; A)$ and $J(X / K ; G ; A) \subseteq J(X / K ; G)$. 
Lemma 2.2. Suppose $(X / K, G / \mathbb{Z}[1 / \Delta], \mathbb{L})$ satisfies (2.2). Suppose that there is a constant $C<1$ such that for each $\ell \in \mathbb{L}$ and each group $H_{\ell}$ of type $G(\mathbb{Z} / \ell)$, $\left|I_{\ell}\left(H_{\ell}\right)\right| /\left|H_{\ell}\right|<C$. Then $J(X / K ; G)$ has density one.

Proof. Let $A \subset \mathbb{L}$ be a finite subset. The Chebotarev density theorem, applied to the representation $\times_{\ell \in A} \rho_{X / K, \ell}$ of $\operatorname{Gal}(K)$, shows that the density of $I(X / K ; G ; A)$ is $\prod_{\ell \in A}\left|I_{\ell}\left(H_{X / K, \ell}\right)\right| /\left|H_{X / K, \ell}\right|$, which is less than $C^{|A|}$. By taking $A$ arbitrarily large, we find that $I(X / K ; G)$ has density zero and its complement, $J(X / K ; G)$, has density one.

Recall that if $G$ is of type (C), or of type A with $r$ odd, and if $\ell$ is inert in $Z$, then the natural representation of $G(\mathbb{Z} / \ell)$ is an irreducible module over $T_{\ell}^{\text {an }}$. Equivalently, some semisimple element of $G(\mathbb{Z} / \ell)$ acts irreducibly on $V \otimes \mathbb{Z} / \ell$.

Lemma 2.3. Suppose $(X / K, G / \mathbb{Z}[1 / \Delta], \mathbb{L})$ satisfies $(2.2)$. Suppose $\mathfrak{p} \in M(X / K)$, and let $\sigma_{\mathfrak{p}}$ be a Frobenius element at $\mathfrak{p}$. If $\rho_{X / K, \ell}\left(\sigma_{\mathfrak{p}}\right) \in J_{\ell}(G)$, and if some semisimple element of $G(\mathbb{Z} / \ell)$ acts irreducibly on $X_{\ell}$, then the reduction $X_{\mathfrak{p}}$ is simple.

Proof. Let $\ell$ be a rational prime relatively prime to $\mathfrak{p}$, and suppose $\rho_{X / K, \ell}\left(\sigma_{\mathfrak{p}}\right) \in$ $J_{\ell}(G)$. Then the group $\left\langle\rho_{X / K, \ell}\left(\sigma_{\mathfrak{p}}\right)\right\rangle$ acts irreducibly on $X_{\ell}$, so that $\left\langle\rho_{X / K, \mathbb{Z}_{\ell}}\left(\sigma_{\mathfrak{p}}\right)\right\rangle$ acts irreducibly on $T_{\ell}(X)$. The Tate module of $X$ is an irreducible Gal $(\kappa(\mathfrak{p}))$-module, thus the abelian variety $X_{\mathfrak{p}} / \kappa(\mathfrak{p})$ is simple [25, Thm. 1(b)].

Lemma 2.4. Suppose $(X / K, G / \mathbb{Z}[1 / \Delta], \mathbb{L})$ satisfies 2.2 , and that $G$ is of type $(\mathrm{A}$ with $r$ even. Suppose $\ell \in \mathbb{L}$ is inert in $Z$, and that $\mathfrak{p} \in M(X / K)$. If $\sigma_{\mathfrak{p}}$ is a Frobenius element at $\mathfrak{p}$, if $\rho_{X / K, \ell}\left(\sigma_{\mathfrak{p}}\right) \in J_{\ell}(G)$, and if $X_{\ell}$ is the natural representation of $G(\mathbb{Z} / \ell)$, then the reduction $X_{\mathfrak{p}}$ is simple.

Proof. Possibly after conjugating, assume that $t:=\rho_{X / K, \ell}\left(\sigma_{p}\right)$ lies in $T_{\ell}^{\text {an }}$. Recall that $T_{\ell}^{\text {an }}$ stabilizes two maximal isotropic subspaces $W_{1}$ and $W_{2}$ of $X_{\ell}$ which are in duality with each other; the action of $T_{\ell}^{\text {an }}$ on $W_{2}$ is the Frobenius twist of its action on $W_{1}$. By Tate's theorem, either $X_{\mathfrak{p}}$ is irreducible, or $X_{\mathfrak{p}} \sim Y_{1} \times Y_{2}$ with each $Y_{j}$ irreducible. In the latter case, the polarization would place $Y_{1}$ and $Y_{2}$ in duality, and in particular $Y_{1}$ and $Y_{2}$ are isogenous. However, since $t$ is a regular element of $T_{\ell}^{\mathrm{an}}$, its eigenvalues on $W_{1}$ are distinct from its eigenvalues on $W_{2}$. Therefore, $X_{\mathfrak{p}}$ is irreducible.

Lemma 2.5. Suppose $(X / K, G / \mathbb{Z}[1 / \Delta], \mathbb{L})$ satisfies $(2.2)$. Suppose that there is a constant $C<1$ such that for each $\ell \in \mathbb{L}$ and each group $H_{\ell}$ of type $G(\mathbb{Z} / \ell)$, $\left|I_{\ell}\left(H_{\ell}\right)\right| /\left|H_{\ell}\right|<C$. Suppose that for each $\ell \in \mathbb{L}$, either

(a) some semisimple element of $G(\mathbb{Z} / \ell)$ acts irreducibly on $X_{\ell}$; or

(b) $G$ is of type A, $r$ is even, $\ell$ is inert in $Z$, and $X_{\ell}$ is the natural representation.

Then $S(X / K)$ has density one.

Proof. Part (a) follows immediately from Lemmas 2.2 and 2.3 Part (b) follows from Lemmas 2.2 and 2.4 . 
In the other direction, we have:

Lemma 2.6. Suppose $X / K$ is an absolutely simple abelian variety over a number field, and suppose $\mathfrak{p} \in M(X / K)$. Suppose that there exist a prime $\ell$ relatively prime to $\mathfrak{p}$, an integer $d \geq 2$, and a $\mathbb{Q}_{\ell}$-representation $W_{\mathbb{Q}_{\ell}}$ of $\operatorname{Gal}(K)$ with $T_{\ell}(X) \otimes \mathbb{Q} \cong W_{\mathbb{Q}_{\ell}}^{\oplus d}$ as $\operatorname{Gal}(K)$-module.

(a) There are simple abelian varietes $Y_{1}, \ldots, Y_{s}$ over $\kappa(\mathfrak{p})$ such that

$$
X_{\mathfrak{p}} \sim Y_{1}^{e_{1}} \times \cdots \times Y_{s}^{e_{s}}
$$

For each $j$ with $1 \leq j \leq s, d \mid e_{j} \cdot d\left(Y_{j}\right)$.

(b) If the residue field $\kappa(\mathfrak{p})$ is a field of prime order, then each $e_{j} \geq d$. In particular, $X_{\mathfrak{p}}$ is not simple.

Proof. Recall that $f_{\mathfrak{p}}(t)$, the characteristic polynomial of Frobenius of $X_{\mathfrak{p}}$, coincides with the characteristic polynomial of $\rho_{X / K, \mathbb{Z}_{\ell}}\left(\sigma_{\mathfrak{p}}\right)$. Since $T_{\ell}(X) \otimes \mathbb{Q} \cong W_{\mathbb{Q}_{\ell}}^{\oplus d}$, there exists a polynomial $g_{\mathfrak{p}, \ell}(t) \in \mathbb{Z}_{\ell}(t)$ with $f_{\mathfrak{p}}(t)=g_{\mathfrak{p}, \ell}(t)^{d}$. Note that $f_{\mathfrak{p}}$ and $g_{\mathfrak{p}, \ell}$ are both monic. By inductively analyzing the coefficients of $g_{\mathfrak{p}, \ell}(t)$ (in descending order), one sees that $g_{\mathfrak{p}, \ell}(t) \in \mathbb{Q}[t] \cap \mathbb{Z}_{\ell}[t] \subset \mathbb{Q}_{\ell}[t]$; by Gauss's lemma, $g_{\mathfrak{p}, \ell}(t) \in \mathbb{Z}[t]$. Factor $g_{\mathfrak{p}, \ell}(t)=g_{1}(t)^{a_{1}} \cdots g_{s}(t)^{a_{s}}$ as a product of powers of distinct irreducible polynomials, so that $f_{\mathfrak{p}}(t)=g_{1}(t)^{a_{1} d} \cdots g_{s}(t)^{a_{s} d}$. Consider some $j$ with $1 \leq j \leq s$. By the theory developed by Tate and Honda [25, Thm. 1(b) and Thm. 2(e)] [26, Thm. 1 and Rem. 2], there is a simple abelian variety $Y_{j}$ over $\kappa(\mathfrak{p})$ with characteristic polynomial of Frobenius $g_{j}(t)^{d\left(Y_{j}\right)}$. Moreover, any abelian variety over $\kappa(\mathfrak{p})$ with characteristic polynomial divisible by $g_{j}(t)$ contains a sub-abelian variety isogenous to $Y_{j}$. From this the decomposition (2.3) follows, where $e_{j}=\frac{a_{j} d}{d\left(Y_{j}\right)}$. This proves (a).

For (b), a simple abelian variety over a prime field has commutative endomorphism ring. (This follows from [26, Thm. 1(ii)], and was noted in [6, p. 469].) Therefore, each $Y_{j}$ has commutative endomorphism ring; $d\left(Y_{j}\right)=1$; and each exponent $e_{j}$ in 2.3 is a multiple of $d \geq 2$.

Lemma 2.7. Suppose $(X / K, G / \mathbb{Z}[1 / \Delta], \mathbb{L})$ satisfies $(2.2)$, where $X$ is an absolutely simple abelian variety. Suppose there is a constant $C<1$ such that for each $\ell \in \mathbb{L}$ and each group $H_{\ell}$ of type $G(\mathbb{Z} / \ell),\left|I_{\ell}\left(H_{\ell}\right)\right| /\left|H_{\ell}\right|<C$. Suppose there is an integer $d \geq 2$ such that for each $\ell \in \mathbb{L}$ there exists an irreducible $\operatorname{Gal}(K)$-module $W_{\mathbb{Q}_{\ell}}$ with $T_{\ell}(X) \otimes \mathbb{Q} \cong W_{\mathbb{Q}_{\ell}}^{\oplus d}$. Finally, suppose there exists $\sigma \in \operatorname{Gal}(K)$ such that $\rho_{X / K, \mathbb{Z}_{\ell}}(\sigma)$ acts irreducibly and semisimply on $W_{\mathbb{Q}_{\ell}}$. Then for $\mathfrak{p}$ in a set of density one there exists a simple abelian variety $Y_{\mathfrak{p}} / \kappa(\mathfrak{p})$ such that $X_{\mathfrak{p}}$ is isogenous to $Y_{\mathfrak{p}}^{\oplus d}$.

Proof. Suppose $\mathfrak{p} \in M(X / K)$, and choose $\ell \in \mathbb{L}$ prime to $\mathfrak{p}$. Let $g_{\mathfrak{p}, \ell}(t) \in \mathbb{Z}[t]$ be the characteristic polynomial of $\sigma_{\mathfrak{p}}$ acting on $W_{\mathbb{Q}_{\ell}}$ via $\rho_{X / K, \mathbb{Z}_{\ell}}$, and let $f_{\mathfrak{p}}(t)$ be the characteristic polynomial of Frobenius of $X_{\mathfrak{p}}$. We have seen (Lemma 2.6) that $f_{\mathfrak{p}}(t)=g_{\mathfrak{p}, \ell}(t)^{d}$.

By Lemma 2.2, $J(X / K ; G)$ has density one. If $\mathfrak{p} \in J(X / K ; G)$, then $\sigma_{\mathfrak{p}}$ acts irreducibly on $W_{\mathbb{Q}_{\ell}}$, and thus $g_{\mathfrak{p}, \ell}(t)$ is irreducible (over $\mathbb{Q}$ ). Therefore, for such $\mathfrak{p}$, $s=1$ in 2.3 , and $X_{\mathfrak{p}} \sim Y^{e}$ for some $e$ with $d(Y) \cdot e=d$.

If we further restrict $\mathfrak{p}$ to have residue degree one (which is still a density-one condition), then $d(Y)=1$ and $e=d$ (Lemma 2.6(b)). 
In fact, we will need slightly stronger variants of Lemmas 2.5 and 2.6 .

Proposition 2.8. Let $X / K$ be an absolutely simple abelian variety over a number field, and let $K^{\prime} / K$ be a finite Galois extension of degree $m$ such that $\left(X / K, K^{\prime}\right)$ satisfies (2.1). Suppose $\left(X / K^{\prime}, G / \mathbb{Z}[1 / \Delta], \mathbb{L}\right)$ satisfies 2.2. Suppose that there is a constant $C<1$ such that for all $\ell \in \mathbb{L}$ and each $H_{\ell}$ of type $G(\mathbb{Z} / \ell),\left|I_{\ell, m}\left(H_{\ell}\right)\right| /\left|H_{\ell}\right|<$ $C$. Suppose that for each $\ell \in \mathbb{L}$, either

(a) some semisimple element of $G(\mathbb{Z} / \ell)$ acts irreducibly on $X_{\ell}$; or

(b) $G$ is of type A, $r$ is even, $\ell$ is inert in $Z$, and $X_{\ell}$ is the natural representation.

Then $S^{*}(X / K)$ has density one.

Proof. We indicate how to prove Lemmas 2.2 and 2.3 in this more general setting. This will prove Proposition 2.8 under hypothesis (a); the result for hypothesis (b) is entirely analogous. Let $B=\operatorname{Gal}\left(K^{\prime} / K\right)$, and for each $\ell$ let $B_{\ell}=H_{X / K, \ell} / H_{X / K^{\prime}, \ell}$. Then $B_{\ell}$ is a quotient of $B$.

Let $J_{m}(X / K ; G)$ be the set of primes $\mathfrak{p} \in M(X / K)$ for which there exists some $\ell \in \mathbb{L}$ such that $\rho_{X / K, \ell}\left(\sigma_{\mathfrak{p}}\right)^{m} \in J_{\ell}(G)$, i.e., such that $\rho_{X / K, \ell}\left(\sigma_{\mathfrak{p}}\right) \in J_{\ell, m}\left(H_{X / K, \ell}\right)$. We start by showing that $J_{m}(X / K ; G)$ has density one.

Suppose $A \subset \mathbb{L}$ is a finite set. The hypothesis $(2.2)$, applied to the subgroup $\prod_{\ell \in A} H_{X / K^{\prime}, \ell}$ of $\prod_{\ell \in A} H_{X / K, \ell}$, implies that there is a quotient $B_{A}$ of $B$ such that the image of $\operatorname{Gal}(K)$ under $\times_{\ell \in A} \rho_{X / K, \ell}$ is an extension of $B_{A}$ by $\prod_{\ell \in A} H_{X / K^{\prime}, \ell}$.

Suppose $\ell \in \mathbb{L}$. Since $\left|B_{\ell}\right|$ is bounded independently of $\ell$, by hypothesis there exists a constant $C^{\prime}<1$ such that

$$
\frac{\left|H_{X / K, \ell}-J_{\ell, m}(G)\right|}{\left|H_{X / K, \ell}\right|}<C^{\prime} .
$$

As in Lemma 2.2. this implies that the set $J_{m}(X / K ; G)$ has density one.

Suppose $\mathfrak{p} \in J_{m}(X / K ; G)$ is not one of the finitely many exceptional primes allowed by (2.1), and choose an $\ell$ such that $\rho_{\ell}\left(\sigma_{\mathfrak{p}}\right) \in J_{\ell, m}(G)$. Not only is $X_{\mathfrak{p}}$ simple, but it is absolutely simple. Indeed, let $\mathfrak{p}^{\prime} \in M\left(X / K^{\prime}\right)$ be a prime lying over $\mathfrak{p}$; then $\rho_{\ell}\left(\sigma_{\mathfrak{p}}^{m}\right)$ is a power of the mod- $\ell$ reduction of the Frobenius element of $X_{\mathfrak{p}^{\prime}}=X_{\mathfrak{p}} \times_{\kappa(\mathfrak{p})} \kappa\left(\mathfrak{p}^{\prime}\right)$, and $X_{\mathfrak{p}^{\prime}}$ is simple. Moreover, $\kappa\left(\mathfrak{p}^{\prime}\right)$ contains the field of definition of the $n$-torsion of $X_{\mathfrak{p}}$. Since $X_{\mathfrak{p}}$ is simple over $\kappa\left(\mathfrak{p}^{\prime}\right)$ (Lemma 2.3), it is absolutely simple (by (2.1)).

Since $J_{m}(X / K ; G) \subseteq S^{*}(X / K)$, the set of primes at which $X$ has absolutely simple reduction has density one.

Proposition 2.9. Suppose $X / K$ is an absolutely simple abelian variety over a number field. Suppose that there exist a finite Galois extension $K^{\prime} / K$, an integer $d \geq 2$, and an infinite set of primes $\mathbb{L}$ such that for each $\ell \in \mathbb{L}$ there exists a representation $W_{\mathbb{Q}_{\ell}}$ of $\operatorname{Gal}\left(K^{\prime}\right)$ such that $T_{\ell}(X) \otimes \mathbb{Q}_{\ell} \cong W_{\mathbb{Q}_{\ell}}^{\oplus d}$ as $\operatorname{Gal}\left(K^{\prime}\right)$-module.

(a) For $\mathfrak{p}$ in a set of density at least $1 /\left[K^{\prime}: K\right]$, there exists an abelian variety $Y_{\mathfrak{p}}$ over $\kappa(\mathfrak{p})$ such that $X_{\mathfrak{p}} \sim Y_{\mathfrak{p}}^{\oplus d}$.

(b) Suppose $\left(X / K^{\prime}, G / \mathbb{Z}[1 / \Delta], \mathbb{L}\right)$ satisfies $\left[2.2\right.$, and that $\left(X / K, K^{\prime}\right)$ satisfies (2.1). Suppose there is a constant $C<1$ such that for each $\ell \in \mathbb{L}$ and each group $H_{\ell}$ of type $G(\mathbb{Z} / \ell),\left|I_{\ell}\left(H_{\ell}\right)\right| /\left|H_{\ell}\right|<C$. Suppose there exists $\sigma \in \operatorname{Gal}\left(K^{\prime}\right)$ such that $\rho_{X / K^{\prime}, \mathbb{Z}_{\ell}}(\sigma)$ acts irreducibly and semisimply on 


$$
\begin{aligned}
& W_{\mathbb{Q}_{\ell}} \text {. Then for } \mathfrak{p} \text { in a set of density at least } 1 /\left[K^{\prime}: K\right], X_{\mathfrak{p}} \times \overline{\kappa(\mathfrak{p})} \sim Y_{\overline{\mathfrak{p}}}^{\oplus d} \\
& \text { for an absolutely simple abelian variety } Y_{\overline{\mathfrak{p}}} / \overline{\kappa(\mathfrak{p})} .
\end{aligned}
$$

Proof. Let $T\left(X / K, K^{\prime}\right)$ be the set of primes $\mathfrak{p} \in M(X / K)$ which lie under some $\mathfrak{p}^{\prime} \in M\left(X / K^{\prime}\right)$ with prime residue field. (Note that $T\left(X / K, K^{\prime}\right)$ has density at least $1 /\left[K^{\prime}: K\right]$.) If $\mathfrak{p} \in T\left(X / K, K^{\prime}\right)$, then $X_{\mathfrak{p}^{\prime}}$ is reducible by Lemma 2.6(b). Since $\kappa\left(\mathfrak{p}^{\prime}\right)=\kappa(\mathfrak{p}), X_{\mathfrak{p}}$ is reducible, too. This proves (a).

Now suppose the hypotheses of (b) hold. Let $T^{*}\left(X / K^{\prime}\right)$ be the set of primes $\mathfrak{p}^{\prime} \in M\left(X / K^{\prime}\right)$ such that $X_{\mathfrak{p}^{\prime}}$ is isogenous to $Y_{\mathfrak{p}^{\prime}}^{\oplus d}$ for some simple abelian variety $Y_{\mathfrak{p}^{\prime}} / \kappa\left(\mathfrak{p}^{\prime}\right)$. By hypothesis (2.1), such a $Y_{\mathfrak{p}^{\prime}}$ is actually absolutely simple. By Lemma 2.7. $T^{*}\left(X / K^{\prime}\right)$ has density one; the set of primes of $K$ lying under elements of $T^{*}\left(X / K^{\prime}\right)$ has density at least $1 /\left[K^{\prime}: K\right]$.

\section{Lefschetz groups}

Suppose $X / K$ is an abelian variety whose endomorphism algebra is a (noncommutative) division algebra. In this section we show that the representation of $\operatorname{Gal}(K)$ on $T_{\ell}(X)$ is isomorphic to a several copies of the same representation. The result follows from an analogous description of Lefschetz groups due to Milne, whose treatment [18] we follow here.

Consider a Weil cohomology theory $X \mapsto H^{*}(X)$ with coefficients in a field $k$ of characteristic zero. Examples of such a theory include Betti cohomology (for varieties over $\mathbb{C}$ ) and $\ell$-adic cohomology. If $X$ is an abelian variety, let $V(X)_{k}$ be the dual of its first cohomology group in this cohomology theory. For example, $V(X)_{\mathbb{Q}_{\ell}}=$ $T_{\ell}(X) \otimes_{\mathbb{Z}} \mathbb{Q}$; and if $X$ is a complex abelian variety, then $V(X)_{\mathbb{Q}}$ is its first Betti homology $H_{1}(X(\mathbb{C}), \mathbb{Q})$.

In this context there is a Lefschetz group $\operatorname{Lef}(X)_{k}$, an algebraic group over $k$ which is naturally a subgroup of $\operatorname{GL}\left(V(X)_{k}\right)$. It is the largest subgroup which fixes the (suitably Tate twisted) cohomology classes of cycles on powers of $X$ which are linear combinations of intersections of divisor classes.

Suppose $X$ is a simple abelian variety; recall the conventions surrounding the endomorphism algebra $D(X)=\operatorname{End}(X) \otimes \mathbb{Q}$ introduced in Section 2, Say that $k$ totally splits $D(X)$ if $E(X) \otimes_{\mathbb{Q}} k \cong \oplus_{\tau: E(X) \hookrightarrow k} k \cong k^{\oplus e(x)}$, and if for each $\tau: E(X) \hookrightarrow$ $k$, one has $D(X) \otimes_{E(X), \tau} k \cong \operatorname{Mat}_{d(X)}(k)$.

Lemma 3.1. Let $X$ be an absolutely simple abelian variety. Consider a Weil cohomology theory with coefficients in a field $k$, and suppose that $k$ totally splits $D(X)$. There is a representation $W_{k}$ of $\operatorname{Lef}(X)_{k}$ such that $V(X)_{k} \cong W_{k}^{\oplus d(X)}$ as $\operatorname{Lef}(X)_{k}$ representations.

Proof. Suppose $k$ is algebraically closed. There exists an algebraic group $\widetilde{\operatorname{Lef}}(X)_{k}$ and a natural isomorphism $\iota: \operatorname{Lef}(X)_{k} \cong \oplus_{\sigma: F(X) \hookrightarrow{ }_{k}} \widetilde{\operatorname{Lef}}(X)_{k}$ [18, Sec. 2]. Moreover, there exists a representation $\widetilde{V}_{k}$ of $\widetilde{\operatorname{Lef}}(X)_{k}$ such that, under the isomorphism $\iota, V(X)_{k}$ and $\oplus_{\sigma: F(X) \hookrightarrow k} \widetilde{V}_{k}^{\oplus d(X)}$ are isomorphic representations of $\operatorname{Lef}(X)_{k}$. Then $W_{k}:=\oplus_{\sigma: F(X) \hookrightarrow k} \widetilde{V}_{k}$ is the sought-for decomposition of $V(X)_{k}$ as $\operatorname{Lef}(X)_{k}$ representation. The analysis in [18, Sec. 2] relies only on the fact that the field of coefficients 
totally splits the endomorphism algebra, and thus the result holds under this weaker hypothesis on $k$.

Recall that for an abelian variety $X$ over a number field $K, H_{X / K \text {; } \mathbb{Q}_{\ell}}$ is the Zariski closure of $\rho_{X / K, \mathbb{Z}_{\ell}}(\operatorname{Gal}(K))$ in $\mathrm{GL}\left(V(X)_{\mathbb{Q}_{\ell}}\right)$.

Lemma 3.2. Let $X / K$ be an absolutely simple abelian variety over a number field. Suppose $\mathbb{Q}_{\ell}$ totally splits $D(X)$. Suppose that $H_{X / K ; \mathbb{Q}_{\ell}}$ is connected. There is a representation $W_{\mathbb{Q}_{e}}$ of $\operatorname{Gal}(K)$ such that, as $\operatorname{Gal}(K)$-modules,

$$
V(X)_{\mathbb{Q}_{\ell}} \cong W_{\mathbb{Q}_{\ell}}^{\oplus d(X)} \text {. }
$$

Proof. Fix an embedding $K \hookrightarrow \mathbb{C}$, so that $X$ has a natural structure of complex abelian variety. Associated to $X$ is its Mumford-Tate group $\operatorname{MT}(X)$. It is an algebraic subgroup of $\mathrm{GL}\left(V(X)_{\mathbb{Q}}\right)$, and there is a natural inclusion $\operatorname{MT}(X) \subseteq \operatorname{Lef}(X)_{\mathbb{Q}}$. Since comparison isomorphisms in cohomology furnish isomorphisms of Lefschetz groups, there are thus natural inclusions $\operatorname{MT}(X) \times \mathbb{Q}_{\ell} \subseteq \operatorname{Lef}(X)_{\mathbb{Q}} \times \mathbb{Q}_{\ell} \cong \operatorname{Lef}(X)_{\mathbb{Q}_{\ell}}$. Work of Deligne, Piateskii-Shapiro and Borovoi (see, for example 9, Prop. 2.9 and Thm. 2.11]) shows there is a natural inclusion

$$
H_{X / K ; \mathbb{Q}_{\ell}} \subseteq \mathrm{MT}(X) \times_{\mathbb{Q}} \mathbb{Q}_{\ell} .
$$

(In general, 3.1) holds only for the connected component $H_{X / K ; \mathbb{Q}_{\ell}}^{0}$; the MumfordTate conjecture asserts that (3.1) is actually an equality.) By Lemma 3.1, there exists a representation $W_{\mathbb{Q}_{\ell}} \subseteq V(X)_{\mathbb{Q}_{\ell}}$ such that $V(X)_{\mathbb{Q}_{\ell}} \cong W_{\mathbb{Q}_{\ell}}^{\oplus d(X)}$. Therefore, $V(X)_{\mathbb{Q}_{\ell}} \cong W_{\mathbb{Q}_{\ell}}^{\oplus d(X)}$ as $H_{X / K ; \mathbb{Q}_{\ell}}$-modules, and thus as $\operatorname{Gal}(K)$-modules.

\section{Commutative endomorphism ring}

Theorem 4.1. Let $X / K$ be an absolutely simple abelian variety over a number field. Suppose $F=\operatorname{End}_{\bar{K}}\left(X_{\bar{K}}\right) \otimes \mathbb{Q}$ is a totally real field. If $r=\operatorname{dim} X /[F: \mathbb{Q}]$ is odd then $S^{*}(X / K)$, the set of primes where $X$ has good, absolutely simple reduction, has density one.

Proof. Using Lemma 2.1, choose a finite Galois extension $K^{\prime} / K$ such that $\left(X / K, K^{\prime}\right)$ satisfies 2.1. Let $G=\mathbf{R}_{\mathcal{O}_{F} / \mathbb{Z}} \mathrm{GSp}_{2 r}$, with derived group $G^{\text {der }}=\mathbf{R}_{\mathcal{O}_{F} / \mathbb{Z}} \mathrm{Sp}_{2 r}$. For all $\ell \gg 0$, the derived group of $H_{X / K^{\prime}, \ell}$ is $G^{\operatorname{der}}(\mathbb{Z} / \ell)$ [2, Thm. B] (see also 21] for the case $r=1$ ), so that $H_{X / K^{\prime}, \ell}$ is of type $G(\mathbb{Z} / \ell)$. Moreover, $X_{\ell}$ is the natural representation of $H_{X / K^{\prime}, \ell}$. By Lemmas 1.1 and 1.3 and Proposition $2.8, S^{*}(X / K)=1$.

Theorem 4.2. Let $X / K$ be an absolutely simple abelian variety over a number field. Suppose there is some prime $\ell_{0}$ such that $H_{X / K, \mathbb{Q}_{0}}=\mathrm{GSp}_{2 g}\left(\mathbb{Q}_{\ell_{0}}\right)$. Then $S^{*}(X / K)$ has density one.

Proof. There is always an a priori inclusion $H_{X / K, \mathbb{Q}_{\ell}} \subseteq \mathrm{GSp}_{2 g}\left(\mathbb{Q}_{\ell}\right)$. Since if the Mumford-Tate conjecture is true for $X$ at one prime $\ell_{0}$ it is true at all primes [17 Thm. 4.3], the hypothesis holds for every rational prime $\ell$. A theorem of Larsen [16. Thm. 3.17], combined with Lemma 1.4, implies that for $\ell$ in a set of primes $\mathbb{L}$ of density one, the derived subgroup $H_{X / K, \ell}^{\mathrm{der}}$ of the image of $\rho_{X / K, \ell}$ is $\mathrm{Sp}_{2 g}(\mathbb{Z} / \ell)$. Choose 
an extension $K^{\prime} / K$ such that $\left(X / K, K^{\prime}\right)$ satisfies $\left[2.1\right.$. Then $H_{X / K^{\prime}, \ell}^{\mathrm{der}} \cong \mathrm{Sp}_{2 g}(\mathbb{Z} / \ell)$ for $\ell$ in a set of primes $\mathbb{L}^{\prime} \subseteq \mathbb{L}$ which still has density one. Again, Lemmas 1.1 and 1.3 and Proposition 2.8 show that $S^{*}(X / K)$ has density one.

Remark 4.3. Under the hypotheses of Theorem 4.2 Chai and Oort show that $S^{*}(X / K)$ has positive density [4, Rem. 5.(iv)]. Under the apparently stronger hypothesis that $H_{X / K, \ell}=\operatorname{GSp}_{2 g}(\mathbb{Z} / \ell)$ for all $\ell \gg 0$, Chavdarov shows that $S^{*}(X / K)$ has density one [5, Cor. 6.10].

Let $X / K$ be an absolutely simple abelian variety of dimension $g$ such that $D(X)=$ $\operatorname{End}_{\bar{K}}(X) \otimes \mathbb{Q} \cong E$, a totally imaginary extension of $\mathbb{Q}$. Let $r=2 g /[E: \mathbb{Q}]$. Fix an embedding $E \hookrightarrow \mathbb{C}$; the tangent space $\operatorname{Lie}\left(X_{\mathbb{C}}\right)$ of $X_{\mathbb{C}}$ is a $g$-dimensional vector space over $\mathbb{C}$, and thus a module over $E \otimes_{\mathbb{Q}} \mathbb{C} \cong \oplus_{\tau: E \hookrightarrow \mathbb{C}} \mathbb{C}$. Let $m_{\tau}$ be the $\mathbb{C}$-dimension of the subspace of $\operatorname{Lie}\left(X_{\mathbb{C}}\right)$ on which $E$ acts via $\tau$. For $\tau \in \operatorname{Hom}(E, \mathbb{C})$, let $\bar{\tau}$ denote the composition of $\tau$ with complex conjugation. Then $m_{\tau}+m_{\bar{\tau}}=r$ is independent of the choice of $\tau$.

Vasiu has proved the Mumford-Tate conjecture for $X$, provided that the action of $E$ on $X$ is non-special [27, Thm. 1.3.4]. We defer a full exposition to loc. cit., but note that each of the following is an example of a non-special action [27, 6.2.4]:

(i) $r=4$ or $r$ is prime;

(ii) there exists a $\tau \in \operatorname{Hom}(E, \mathbb{C})$ such that $m_{\tau}=1$;

(iii) there exist $\tau$ and $\tau^{\prime}$ such that $1 \leq m_{\tau}<m_{\tau^{\prime}} \leq r / 2$ and either $\operatorname{gcd}\left(m_{\tau}, r\right)$ or $\operatorname{gcd}\left(m_{\tau^{\prime}}, r\right)$ is 1 ;

(iv) there exists a $\tau$ such that $\operatorname{gcd}\left(m_{\tau}, m_{\bar{\tau}}\right)=1$, and the natural numbers $\left(m_{\tau}, m_{\bar{\tau}}\right)$ are not of the form $\left(\left(\begin{array}{c}i \\ j-1\end{array}\right),\left(\begin{array}{c}i \\ j\end{array}\right)\right)$ for any natural numbers $i$ and $j$.

The case of the Mumford-Tate conjecture where $[E: \mathbb{Q}]=2$ and $r=g$ is prime is due to Chi [8, Cor. 3.2].

Theorem 4.4. Let $X / K$ be an absolutely simple abelian variety over a number field. Suppose $E:=\operatorname{End}_{\bar{K}}\left(X_{\bar{K}}\right) \otimes \mathbb{Q}$ is a totally imaginary field, and that $X$ is of non-special type. Then $S^{*}(X / K)$ has density one.

Proof. Since Murty and Patankar have proved this result for abelian varieties of CM type [20, Thm. 3.1], we assume that $2 \operatorname{dim} X /[E: \mathbb{Q}]>1$. Let $K^{\prime} / K$ be a finite extension such that $\left(X / K, K^{\prime}\right)$ satisfies (2.1). By [27, Thm. 1.3.4], the Mumford-Tate conjecture is true for each representation $\left.\rho_{X / K, \mathbb{Q}_{\ell}}\right|_{\operatorname{Gal}\left(K^{\prime}\right)}$. More precisely, there is a group $G / \mathbb{Z}[1 / \Delta]$ of type $A$ such that for almost all $\ell$, the Zariski closure of $H_{X / K^{\prime}, \mathbb{Q} \ell}$ is isomorphic to a subgroup of $G\left(\mathbb{Q}_{\ell}\right)$ which contains $G^{\text {der }}\left(\mathbb{Q}_{\ell}\right)$. By [16, Thm. 3.17] and Lemma 1.4 there is a set of primes $\mathbb{L}$ of density one such that for $\ell \in \mathbb{L}, H_{X / K^{\prime}, \ell}$ is of type $G(\mathbb{Z} / \ell)$. Moreover, $X_{\ell}$ is the natural representation of $H_{X / K^{\prime}, \ell}$. Since the groups $G(\mathbb{Z} / \ell)$ satisfy Goursat's lemma (Lemma 1.3$), S^{*}(X / K)$ has density one by Lemma 1.1 and Proposition 2.8 .

Via the Torelli functor, these results yield information about curves. For example, consider the following condition on a curve $C$ over a field $k$ :

$$
\text { If } C \rightarrow D \text { is finite of degree at least } 2 \text {, then } D \text { has genus zero. }
$$


If the Jacobian $\operatorname{Jac}(C)$ is simple, then $C$ satisfies 4.1 . The converse is true if the genus of $C$ is at most 6 , since almost every principally polarized abelian variety is a Jacobian in dimension at most 3 .

Corollary 4.5. Let $C / K$ be a curve of genus of odd prime genus $g$ over a number field such that $C / \bar{K}$ satisfies 4.1). Suppose that either $g \in\{3,5\}$ or that $\operatorname{Jac}(C)$ is absolutely simple. For almost all primes $\mathfrak{p}, C_{\mathfrak{p}} / \kappa(\mathfrak{p})$ satisfies 4.1.

Proof. By hypothesis (and the preceding discussion), $\operatorname{Jac}(C)$ is absolutely simple. A simple abelian variety of odd prime dimension over a number field has commutative endomorphism ring. This endomorphism ring is totally real or totally imaginary; and in the latter case, the action is not special. Now use Theorem 4.1 or 4.4 as appropriate.

In general, a curve $C$ which satisfies 4.1 need not have reductions $C_{\mathfrak{p}}$ satisfying (4.1) for a dense, or even infinite, set of primes $\mathfrak{p}$. Indeed, let $C$ be the second curve considered in the introduction. Then $\operatorname{Jac}(C)$ is simple, thus $C$ satisfies 4.1 ; but for each prime $\mathfrak{p}$ of good reduction, $\operatorname{Jac}\left(C_{\mathfrak{p}} \times \overline{\kappa(\mathfrak{p})}\right)$ dominates, and thus $C_{\mathfrak{p}}$ covers, an elliptic curve.

\section{Noncommutative endomorphism ring}

Recall the definitions of $D(X)$ and $d(X)$ from Section 2 .

Proposition 5.1. Let $X / K$ be an absolutely simple abelian variety over a number field. Suppose that $\operatorname{End}_{\bar{K}}\left(X_{\bar{K}}\right)$ is noncommutative.

(a) Then $R(X / K)$, the set of primes $\mathfrak{p}$ such that $X_{\mathfrak{p}}$ is reducible, has positive density.

(b) If $\operatorname{End}_{K}(X)=\operatorname{End}_{\bar{K}}(X)$ and $H_{X / K, \mathbb{Q}_{\ell}}$ is connected, then $R(X / K)$ has density one.

Proof. Let $K^{\prime} / K$ be a finite extension such that $\left(X / K, K^{\prime}\right)$ satisfies (2.1); such an extension exists by Lemma 2.1. The conclusion of (b) for $X_{K^{\prime}}$ implies the conclusion of (a) for $X$. Therefore, it suffices to assume $\operatorname{End}_{K}(X)=\operatorname{End}_{\bar{K}}(X)$ and that $H_{X / K, \mathbb{Q} \ell}$ is connected, and then prove that $R(X / K)=M(X / K)$.

Consider the set $\mathbb{L}$ of primes $\ell$ such that $\mathbb{Q}_{\ell}$ totally splits $D(X)$. Note that $\mathbb{L}$ has positive density, and in particular is infinite. Suppose $\ell \in \mathbb{L}$. By Lemma 3.2, there exists a representation $W_{\mathbb{Q}_{\ell}}$ of $\operatorname{Gal}(K)$ such that $T_{\ell}(X) \otimes \mathbb{Q} \cong W_{\mathbb{Q}_{\ell}}^{\oplus d(X)}$ as $\operatorname{Gal}(K)$ module. Note that $d(X)>1$ since $D(X)$ is noncommutative. By Lemma 2.6, for $\mathfrak{p}$ in a subset of $M(X / K)$ of density one, $X_{\mathfrak{p}}$ is isogenous to $Y_{\mathfrak{p}}^{\oplus d(X)}$ for some abelian variety $Y_{\mathfrak{p}} / \kappa(\mathfrak{p})$.

Remark 5.2. In the special case of an abelian surface $X$ with action by an indefinite quaternion algebra, $X$ has absolutely split reduction at every prime of good reduction. This is explained in detail in [19, Sec. 2]; see also [4, Rem. 5.(ii)]. It is possible that Lemma 5.3 will yield a generalization of this. In the context of Proposition 5.1, Murty and Patankar show [20, Prop. 5.4] that $X_{\mathfrak{p}}$ is not simple at any prime $\mathfrak{p}$ of ordinary reduction. 
Lemma 5.3. Let $X / K$ be an absolutely simple abelian variety over a number field with noncommutative endomorphism algebra $D(X)$. Let $\Delta$ be the product of all (finite) primes of $E(X)$ which ramify in $D(X)$. Suppose $\mathfrak{p} \in M(X / K)$ is relatively prime to $\Delta$. Then $E\left(X_{\mathfrak{p}}\right)$ is ramified at every prime dividing $\Delta$.

Proof. Suppose $X_{\mathfrak{p}}$ is simple, and let $p$ be the characteristic of $\kappa(\mathfrak{p})$. The inclusion $\operatorname{End}(X) \hookrightarrow \operatorname{End}\left(X_{\mathfrak{p}}\right)$ forces $D\left(X_{\mathfrak{p}}\right)$ to be noncommutative. By [25, Thm. 2(e)], $D\left(X_{\mathfrak{p}}\right)$ is split at all primes not dividing $p$. In particular, $D\left(X_{\mathfrak{p}}\right)$ is split at all primes not dividing $\Delta$. Since only a ramified field extension splits a division algebra over a local field, $E\left(X_{\mathfrak{p}}\right)$ must ramify at all primes dividing $\Delta$.

If the endomorphism ring of $X$ is an indefinite quaternion algebra, one knows more about the structure of the reductions $X_{\mathfrak{p}}$ :

Theorem 5.4. Let $X / K$ be an absolutely simple abelian variety over a number field. Suppose that $\operatorname{End}_{\bar{K}}\left(X_{\bar{K}}\right) \otimes \mathbb{Q}$ is an indefinite quaternion algebra over a totally real field $F$. If $\operatorname{dim} X / 2[F: \mathbb{Q}]$ is odd, then for $\mathfrak{p}$ in a set of positive density, $X_{\mathfrak{p}}$ is geometrically isogenous to the self-product of an absolutely simple abelian variety $Y_{\overline{\mathfrak{p}}} / \overline{\kappa(\mathfrak{p})}$ of dimension $(\operatorname{dim} X) / 2$.

Proof. Let $K^{\prime} / K$ be a finite extension of $K$ such that $\left(X / K, K^{\prime}\right)$ satisfies 2.1). By [2, Thm. B], Lemma 1.1 and Lemma 1.3 , there exist a group $G / \mathbb{Z}[1 / \Delta]$ of type (C) and an infinite set of primes $\mathbb{L}$ such that $\left(X / K^{\prime}, G / \mathbb{Z}[1 / \Delta], \mathbb{L}\right)$ satisfies $(2.2)$. Moreover, there is a $G$-module $W / \mathbb{Z}[1 / \Delta]$ such that for all $\ell \in \mathbb{L}, W \otimes \mathbb{Z} / \ell$ is an irreducible $G(\mathbb{Z} / \ell)$-module and $X_{\ell} \cong(W \oplus W) \otimes \mathbb{Z} / \ell$ as $G(\mathbb{Z} / \ell)$-module. (This is [2, Thm. 5.4]; see also 7 for the analogous statment for $\mathbb{Q}_{\ell}$-modules.) The result now follows from Proposition 2.9 .

\section{Acknowledgements}

My debt to the work of Murty and Patankar is obvious, and I thank V. Kumar Murty for sharing [20] with me in preprint form. It's also a pleasure to acknowledge helpful discussions with Bob Guralnick, Tim Penttila, Rachel Pries and David Zywina, as well as comments from the referee. The author was partially supported by NSA grant H98230-08-1-0051.

\section{References}

[1] C. Adimoolam, A note on good reduction of simple Abelian varieties, Proc. Amer. Math. Soc. 64 (1977), no. 2, 196-198.

[2] G. Banaszak, W. Gajda, and P. Krasoń, On the image of l-adic Galois representations for abelian varieties of type I and II, Doc. Math. (2006), no. Extra Vol., 35-75 (electronic).

[3] P. Bending, Curves of genus 2 with $\sqrt{2}$ multiplication (2006). ArXiv:math.NT/991723.

[4] C.-L. Chai and F. Oort, A note on the existence of absolutely simple Jacobians, J. Pure Appl. Algebra 155 (2001), no. 2-3, 115-120.

[5] N. Chavdarov, The generic irreducibility of the numerator of the zeta function in a family of curves with large monodromy, Duke Math. J. 87 (1997), no. 1, 151-180.

[6] W. C. Chi, On the l-adic representations attached to some absolutely simple abelian varieties of type II, J. Fac. Sci. Univ. Tokyo Sect. IA Math. 37 (1990), no. 2, 467-484.

[7] - On the Tate modules of absolutely simple abelian varieties of type II, Bull. Inst. Math. Acad. Sinica 18 (1990), no. 2, 85-95. 
[8] - On the l-adic representations attached to simple abelian varieties of type IV, Bull. Austral. Math. Soc. 44 (1991), no. 1, 71-78.

[9] P. Deligne, Hodge cycles on abelian varieties, in Hodge cycles, motives, and Shimura varieties, Vol. 900 of Lecture Notes in Mathematics, 9-100, Springer-Verlag, Berlin (1982), ISBN 3-54011174-3.

[10] J. Ellenberg, C. Elsholtz, C. Hall, and E. Kowalski, Non-simple abelian varieties in a family: geometric and analytic approaches, to appear in the J. London Math. Soc.

[11] P. Fleischmann and I. Janiszczak, The number of regular semisimple elements for Chevalley groups of classical type, J. Algebra 155 (1993), no. 2, 482-528.

[12] T. Ibukiyama, T. Katsura, and F. Oort, Supersingular curves of genus two and class numbers, Compositio Math. 57 (1986), no. 2, 127-152.

[13] J.-i. Igusa, Arithmetic variety of moduli for genus two, Ann. of Math. (2) 72 (1960) 612-649.

[14] E. Kowalski, Weil numbers generated by other Weil numbers and torsion fields of abelian varieties, J. London Math. Soc. (2) 74 (2006), no. 2, 273-288.

[15] T. Kubota, On the field extension by complex multiplication, Trans. Amer. Math. Soc. 118 (1965) 113-122.

[16] M. J. Larsen, Maximality of Galois actions for compatible systems, Duke Math. J. 80 (1995), no. 3, 601-630.

[17] M. J. Larsen and R. Pink, Abelian varieties, l-adic representations, and l-independence, Math. Ann. 302 (1995), no. 3, 561-579.

[18] J. S. Milne, Lefschetz classes on abelian varieties, Duke Math. J. 96 (1999), no. 3, 639-675.

[19] V. K. Murty, Splitting of abelian varieties: a new local-global problem, in Algebra and number theory, 258-268, Hindustan Book Agency, Delhi (2005).

[20] V. K. Murty and V. M. Patankar, Splitting of abelian varieties, IMRN 2008 (2008) Art. ID rnn033, 27 pages.

[21] K. A. Ribet, Galois action on division points of Abelian varieties with real multiplications, Amer. J. Math. 98 (1976), no. 3, 751-804.

$[22] —$ Division fields of abelian varieties with complex multiplication, Mém. Soc. Math. France (N.S.) (1980/81), no. 2, 75-94. Abelian functions and transcendental numbers (Colloq., Étole Polytech., Palaiseau, 1979).

[23] A. Silverberg, Fields of definition for homomorphisms of abelian varieties, J. Pure Appl. Algebra 77 (1992), no. 3, 253-262.

[24] A. Silverberg and Y. G. Zarhin, Connectedness extensions for abelian varieties, Math. Z. 228 (1998), no. 2, 387-403.

[25] J. Tate, Endomorphisms of abelian varieties over finite fields, Invent. Math. 2 (1966) 134-144.

[26] — Classes d'isogénie des variétés abéliennes sur un corps fini (d'après T. Honda), in Séminaire Bourbaki, 352, 95-110 (1968).

[27] A. Vasiu, Some cases of the Mumford-Tate conjecture and Shimura varieties, Indiana Univ. Math. J. 57 (2008) 1-76.

[28] D. Zywina, The large sieve and Galois representations, Ph.D. thesis, University of California, Berkeley (2008).

E-mail address: j.achter@colostate.edu

Department of Mathematics, Colorado State University, Fort Collins, CO 80523-1874

URL: http://www . math. colostate.edu/ achter 